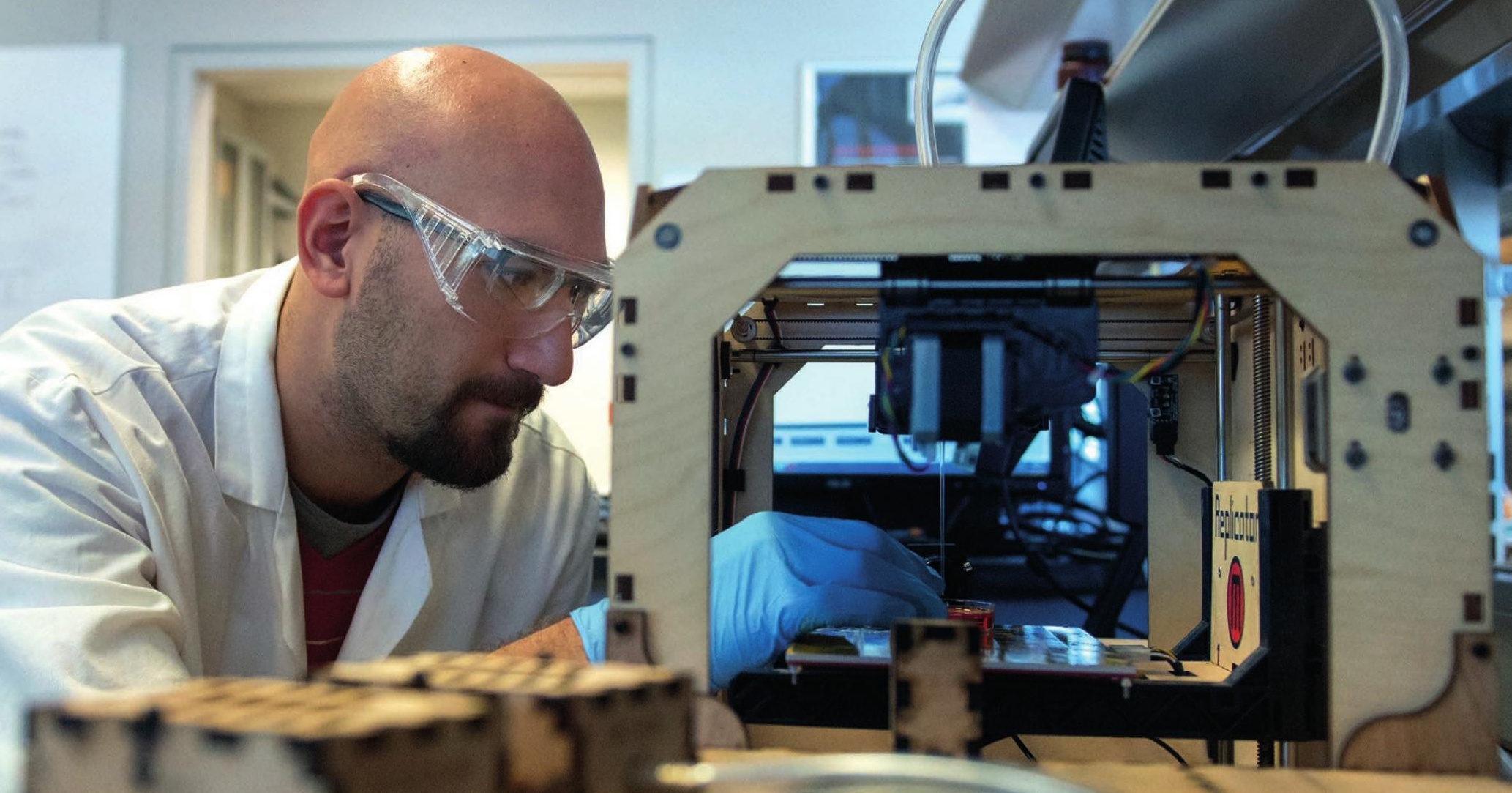

A tissue model is bioprinted using a 3D printer at Pennsylvania State University in University Park.

TECHNOLOGY

\title{
The promise of printing
}

\section{Machines that deposit cell-laden inks are constructing tissues and organs, one layer at a time.}

\section{BY NEIL SAVAGE}

$\mathrm{P}$ eople in their 30 s are probably not thinking about needing surgery to repair their knees. But in a few decades, the rigours of daily living or the ravages of arthritis may send them to the operating theatre. And there, they might find a 3D printer ready to build new bone or cartilage in their bodies. Such is the hope of Ibrahim Ozbolat, a biological engineer at Pennsylvania State University in University Park who is developing 3D-printing techniques to repair tissue such as cartilage. $\mathrm{He}$ envisions a machine that will deposit successive layers of biocompatible material, laden with cells, into a defect. "In the future, we can have the patient under the bioprinter," says Ozbolat. And his vision is not limited to knees. "Whatever section of the body needs to be fixed, the bioprinter can repair that."

Many share Ozbolat's goal of producing living tissue in carefully designed shapes to repair or replace damaged parts of the body. As well as bone and cartilage, researchers are trying to develop skin, nervous-system tissue such as retinas, and even organs such as kidneys or a heart that would be printed externally and then transplanted into the body. But it may be a decade or more before even simple constructs make their way into clinical use. Before something as complex as a kidney is possible, researchers will need to refine the materials, the implantation techniques and their ability to create complex structures such as vasculature. If $3 \mathrm{D}$ printing can be used to produce tissue on demand, it would revolutionize medicine, creating readily available transplant organs and tissues and eliminating the waiting list for replacement parts.

\section{AN ENGINEERING MARVEL}

In the engineering world, $3 \mathrm{D}$ printing is an umbrella term for a range of technologies that construct objects of almost any design by depositing layers of material. Working from a computer pattern, 3D printers can build complex objects that wouldn't be possible with more-conventional techniques, creating, for instance, internal contours where no tool could reach. They have become popular for generating quick prototypes, and are finding their way into the manufacturing processes of everything from medical device to aerospace industries.

The biological version is called $3 \mathrm{D}$ bioprinting, and it uses many of the same techniques, but adjusted to handle living cells. Often, the cells are contained within hydrogels - soft, jelly-like polymers that hold large amounts of water, but are viscous enough to hold their shape, at least for a while. The cells can also be suspended in a solution, like particles of pigment in printer ink.
The bioinks are sprayed through inkjet nozzles or extruded onto a base. They can be solidified by a change in temperature or pressure, or by adding chemicals or a wavelength of light. Once the right structure has been created, nutrients and growth factors are added to encourage the cells to develop into the right kind of tissue.

Researchers are testing combinations of techniques for printing a wide range of tissue types. Bone, for instance, grows best with a scaffold of cell-laden hydrogel, which orients the cells and gives them cues on where to grow. But cartilage, Ozbolat says, grows best when the cells determine their own arrangement, the way they do during embryonic development; a scaffold hinders the process. Cells within one hydrogel don't communicate well with those in another, and so don't provide the signals that cause them to grow together into a larger piece of tissue.

Ozbolat has developed a bioink based on alginate, a seaweed extract. He extruded thin strands of the ink containing cartilage cells, and these grew together, forming a large piece of tissue ${ }^{1}$. He plans to use this technique with pancreatic cells to print islets of Langerhans, the insulin-producing parts of the pancreas, for transplantation into people with type 1 diabetes. And his technology works in living animals: by adding collagen to the bioink, he built bone and skin in the skull wounds of rats. 
Bioprinted cartilage could prove to be superior to both donor tissue and the plastic and titanium used in knee replacements, says Darryl D'Lima, director of orthopaedic research at the Scripps Clinic in San Diego, California. Although knee replacements made of plastic and metal last for 20 years, they're not as resilient as living tissue. "Metal and plastic and cement are strongest the day after you put them in," D'Lima says. "After that, they will only get weaker." And printed cartilage may turn out to be stronger than grafts from donated tissue. Not only can tissue be printed in the shape of the damage, avoiding the need to cut away healthy tissue, but the bioink should also fill the tiny crevices that no surgery could address, leading to greater overall strength. He admits that this is speculation; the technology isn't ready for comparative clinical trials. Researchers such as D'Lima are experimenting with 3D-printed parts in lab animals, but haven't yet proved that they're superior to conventional grafts.

D'Lima has been investigating 3D printing for the eyes, with the aim of treating the blindness caused by deterioration of the retina as it ages. He and Jeffrey Goldberg, an ophthalmologist at Stanford University in California, printed retinal ganglion cells onto scaffolding to see if they could grow retinas ${ }^{2}$. Because the ganglion cells are neurons, they need to grow in a certain orientation. "We want their axons to point and grow in a particular direction because we want to get them to the right target," Goldberg says. The axons need to reach along the optic nerve to connect the retina to the brain. D'Lima and Goldberg managed this by creating a scaffold with a radial pattern that mimics the direction of the nerve fibres in the eyes, then printing the cells along those radial lines. Hydrogels that were rich in the fibrous protein laminin and contained some alignate helped to anchor the retinal cells in place, so that their natural signalling mechanisms could encourage them to grow in the right orientation. The researchers created structures with $72 \%$ of the axons oriented radially, compared with only $11 \%$ of those cultured in $2 \mathrm{D}$ on a plate.

One of the advantages of bioprinting for growing retinas is the specificity it affords. The retina contains two types of photoreceptor: rods and cones. Rods are more concentrated at the edge of the retina, whereas there are more cones in the centre. Bioprinting provides fine control over their placement. "You can't do that with any other technology," Goldberg says. It will probably be a few years before printed retinas can be tried as transplants, he says. One challenge is that nerve cells are packed more densely than, say, cartilage cells, and researchers are not yet sure how to achieve that density.

One line of research involves finding the best bioinks. Different materials have unique properties that affect whether they are easy to print with or hold their shape well, and whether they encourage cell growth. An ideal ink would stay liquid, so it can be easily dispensed, but quickly turn into a more solid, gel-like structure without the use of chemicals or heavy doses of radiation that can harm the cells. "If you look at the technology of bioprinting, probably the limitation at the moment is the availability of bioinks," says Adam Perriman, a chemist at the University of Bristol, UK. Perriman has created a bioink that is a mixture of the hydrogel Pluronic (a mix of poloxamers) and alginate. This mix allows him to fine-tune the gelling time - Pluronic structures hold their shape well, but melt away too easily with changes in temperature, whereas alginate can gel too quickly. The mixed ink allows Perriman to print the structure he wants, then, once it's solidified, wash away the Pluronic, with the bonus that the gel leaves behind a network of micropores that allow the printed tissue to

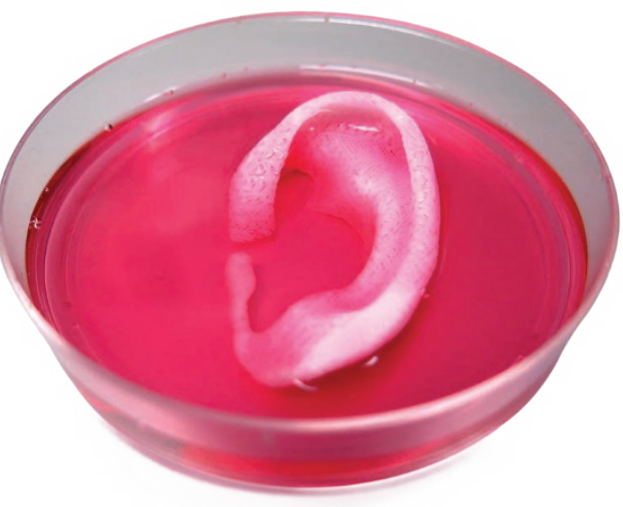

Ear-shaped cartilage could be one of the first bioprinted tissues in clinical use.

take up nutrients ${ }^{3}$.

In another approach, Dong-Woo Cho, a mechanical engineer at Pohang University of Science and Technology in Gyeongbuk, South Korea, is using extracellular matrix to create bioink. He takes heart tissue, cartilage or fat and washes away all trace of cells, leaving behind just extracellular matrix, which consists of materials such as collagen and glycosaminoglycans ${ }^{4}$. He grinds up that material and mixes it with acetic acid and sodium hydroxide to make an ink that can be extruded in filaments, which gel when they're heated to body temperature. Cho thinks that, because the bioink is made from the matrix in which cells live, it will provide a more natural environment for the cells to thrive, and that it will be more biocompatible than inks made from materials not usually found in the body. He's using it to develop patches that could be helpful in the repair of heart damage.

Anthony Atala, director of the Wake Forest Institute for Regenerative Medicine in WinstonSalem, North Carolina, thinks that some simpler 3D-printed tissues could be in clinical use within the next few years. The first type to become available will probably be cartilage it is a relatively flat structure that contains few cell types and does not require a blood supply. Atala has already printed cartilage and bone, and transplanted them into mice. In humans, cartilage could be followed by hollow tubes such as an artery or urethra, and then hollow organs such as a bladder. "Of course, all tissues are complex, but the least complex are flat structures such as skin," Atala says.

A solid organ, however, may have a dozen or more cell types, and would need a vascular system to carry nutrients to its cells. Cells more than around 200 micrometres - a couple of human-hair widths - away from a source of nutrients quickly die, so creating a realistic vascular system will be necessary if researchers are to successfully build organs.

A team at Harvard University in Cambridge, Massachusetts, has taken the first steps to overcome that hurdle, printing thick tissue with a rudimentary vascular system and keeping it alive for weeks ${ }^{5}$. The team used three different inks: silicone to give a basic shape; a bioink infused with pluripotent stem cells that would turn into the tissue; and Pluronic, which is a gel at room temperature, but a liquid when cooled. The team printed the tissue, using the Pluronic to create threads throughout the tissue; after printing, the structure was cooled to $4{ }^{\circ} \mathrm{C}$, then the liquid was drained off to leave behind channels through which nutrients could flow.

Using this process, the Harvard team printed tissue one centimetre thick and kept it alive for more than six weeks. This was long enough for the stem cells to establish a bottom membrane of calcium phosphate, the base on which to grow bone. "There's really no limitation. We could go thicker," says Jennifer Lewis, the bioengineer who led the research. The vasculature in this case was rudimentary, just a set of crisscrossing channels in successive layers. Actual organs, she says, will require a more complex pattern of veins and capillaries of varying sizes.

And bioprinting has the potential to do more than replace body parts with equivalent organs. Ozbolat is thinking about the prospect of enhancing the human body with new types of tissue. For instance, his lab has taken some preliminary steps towards creating an organ that converts chemical energy into electricity - a human version of what an electric eel can do. A person could have his or her own built-in, rechargeable battery to run anything from a pacemaker to a prosthetic limb. Even without printing body parts to give people superhuman abilities, bioprinting holds the promise of writing a new chapter in medicine. How many lives may be saved or bodies improved when it becomes possible to print an unlimited supply of replacement parts?

Neil Savage is a freelance science writer in Lowell, Massachusetts.

1. Yu, Y. et al. Sci. Rep. 6, 28714 (2016).

2. Kador, K. E. et al. Tissue Eng. A 22, 286-294 (2016)

3. Armstrong, J. P. K., Burke, M., Carter, B. M., Davis, S. A. \& Perriman, A. W. Adv. Healthc. Mater. 5, 1724-1730 (2016)

4. Pati, F. et al. Nature Commun. 5, 3935 (2014).

5. Koleskya, D. B., Homana, K. A., Skylar-Scotta, M. A. \& Lewis, J. A. Proc. Natl Acad. Sci. USA 113, 3179-3184 (2016). 